\title{
Journal of Intelligent Industrial Systems: Preface to Vol. 2, No. 2
}

\author{
G. Rigatos $^{1}$ - P. Siano ${ }^{2}$
}

Published online: 24 June 2016

(C) Springer Science+Business Media Singapore 2016

The new issue of the Journal of Intelligent Industrial Systems is structured round two axes: (i) control of dynamical systems exhibiting nonlinear dynamics, and (ii) reliability indexes for the assessment of the performance of production systems. Regarding (i), the first control problem treated in the issue is that of fuel cells for electric power generation. The problem is solved with the use of global linearization methods which finally enable the transformation of the model into an equivalent linear form and the application of stabilizing feedback control. The second control problem treated in the issue is that of pointwise control of distributed parameters systems, that is of dynamical systems exhibiting spatio-temporal variations and being described by PDEs. The third control problem treated in the issue is related to the temperature dynamics of dry dual clurch. A practical solution is developed based on multiple PID loops and the associated stability problem remains open for further investigation. Regarding (ii) the first problem treated is that of definition of indexes that enable to describe and assess the performance of systems. In the same direction the second problem treated is the modelling and assessment of the performance of production units. Both the exploitation of human experience and the automated knowledge extracted through data processing are considered. Finally the issue hosts an article on the problem of reliable hand gesture recognition through elaborated infor-

\footnotetext{
G. Rigatos

grigat@ieee.org

P. Siano

psiano@unisa.it

1 Unit of Industrial Automation, Industrial Systems Institute, 26504 Rion Patras, Greece

2 Department of Industrial Engineering, University of Salerno, 84084 Fisciano, Italy
}

mation processing techniques. All topics described above are of high interest and of direct application to industry.

In article "A PEM fuel cells control approach based on differential flatness theory" by G. Rigatos and P. Siano, an approach s developed for nonlinear control of fuel cells using differential flatness theory and Kalman filtering. First, it is proven that the dynamic model of fuel cells is a differentially flat one which means that all its state variables and control inputs can be expressed as differential functions of specific stare variables which are the so-called flat outputs of the system. By exploiting the differential flatness properties of the model its transformation to an equivalent linear form (canonical Brunovsky form) becomes possible. For the latter description of the systems dynamics the design of a statefeedback controller is achieved. This control scheme should be also robust to model uncertainties and external perturbations. To cope with this problem the state-space description of the PEM fuel cells is extended by considering as additional state variables the derivatives of the aggregate disturbance input. Next, a Kalman Filter-based disturbance observer is applied to the linearized extended model of the fuel cells. This estimation method enables to identify the disturbance and model uncertainty terms that affect the system and to introduce a complementary control element that compensates for the perturbations effects. The efficiency of the proposed control scheme is evaluated through simulation experiments.

In article "Control of the nonlinear wave-type dynamics using the Derivative-free nonlinear Kalman Filter" by G. Rigatos and $\mathrm{P}$, Siano, the objective is to develop a pointwise control method for a 1D nonlinear wave equation and a filtering approach for estimating the dynamics of such a system from measurements provided from a small number of sensors. It is shown that the numerical solution of the associated partial differential equation results into a set of nonlinear ordinary differential equations. With the application of a dif- 
feomorphism that is based on differential flatness theory it is shown that an equivalent description of the system in the linear canonical (Brunovsky) form is obtained. This transformation enables to obtain estimates about the state vector of the system through the application of the standard Kalman Filter recursion. For the local subsystems, into which the nonlinear wave equation is decomposed, it becomes possible to apply pointwise state estimation-based feedback control. The efficiency of the proposed filtering and control approach for nonlinear systems described by 1D partial differential equations of the wave type (e.g. sine-Gordon PDE) is confirmed through simulation experiments. It is shown that, by applying feedback control, the nonlinear wave-type dynamics can be made to track any reference setpoint.

In article "Dry Dual Clutch Torque Model with Temperature and Slip Speed Effects" the problem of modelling and control of the temperature's dynamic model of dry dual clutches is analyzed. Dry dual clutches are widely used in automated transmissions. In such systems the clutch engagement maneuvers require a precise knowledge of the characteristics that relate the frictional torque transmitted by the clutches with the corresponding actuators variables. In this paper a temperature and slip speed dependent model of the torque characteristic for dry dual clutches is proposed. Dynamic models of the temperature evolution are determined and linked to the characteristics of the mechanical components influencing the torque. The models, whose parameters are tuned with dedicated experiments and realistic data coming from an industrial automotive environment, show the temperature influence on the torque transmitted by the clutch. Real time simulation results, obtained through a detailed software in the loop driveline model, show that, if not compensated, the temperature variation can determine critical degradations of the clutch engagement performances. It is shown how the use of the clutch temperature estimation in the torque transmissibility model allows to compensate for such negative effects. The torque model is also exploited for the realization of a decoupling clutch engagement controller. The corresponding closed loop results show the effectiveness of the proposed compensations for the dependencies of the clutch torque on temperature and slip speed. The stability issues of the control scheme remain an issue for further investigation.

In article "Unified Reliability Index Development for Utility Performance Assessment" by H. Sindi, the problem of reliability indices is analyzed as an approach to assessing a system's performance. It is explained that the lack of consensus among utilities and regulators on which indices should be used complicates further this problem. Regulators inevitably make comparisons between utilities' which are routinely reported as reliability indices. Thus, an adequate and fair process needs to be implemented.In this work, a development of a unified reliability index is pursued, aiming at proper per- formance assessment, fair comparisons, and reflection of all the knowledge embedded within all current indices. The initiated unified index may provide several benefits, among which is suitable standards design, improved tools for planning and design optimization, and less technical burden on operators. Also, the development of a unified reliability index requires the initiation of a standard normalization methodology based on a maximum that takes into account the variation between systems under study. After normalization, it is shown thaat the combination of indices is made possible through a proposed methodology. The developed methodology is validated by comparing it to both simple averaging technique and a fuzzy inference based engine.

In article "Automated determining of manufacturing properties and their evolutionary changes from event traces" by J.Ladiges, the problem of modelling and assessment of the performance of production units is analyzed. Production plants are usually kept in operation for several decades. During this long operational phase operation requirements and other production conditions change frequently. Accordingly, the plants have to be adjusted in behavior and/or structure by adapting software and physics of the plant to avoid degeneration. Unfortunately, in industrial practice, changes, especially smaller ones, are often performed ad-hoc without appropriate adaptation of formal models or documentation. As a consequence, knowledge about the process is only implicitly available and an evaluation of performed changes is often omitted, resulting in sub-optimal production performance. Present research approaches to overcome these deficiencies usually concentrate on (a) manual modelling with manual or automatic analysis on a high level of abstraction; or (b) on automatic model generation from observations without lifting gathered knowledge to easy interpretable indicators. The approach presented in this paper combines both methods (a) and (b) by learning models from observation of input output signals of the production plant's control system. Semantics are added by using a priori information modelling which is less tedious compared to modelling the process itself. The learned models are used to automatically detect changes by continuously comparing their behavior with real plant behavior during operation as well as to evaluate performed changes. An analysis of the models results in high-level property values such as key performance indicators or flexibility measures of the production system.

In article "Human Computer Interaction for Vision-Based Hand Gesture Recognition: A Review" an outline is provided about the problem of human computer interaction for vision-based hand gesture recognition. Considerable effort has been put toward the development of intelligent and natural interfaces between users and computer systems. In line with this endeavor, several modes of information (e.g., visual or audio) that are used either individually or in combination have been proposed. The use of gestures to convey infor- 
mation is an important part of human communication. Hand gesture recognition is widely used in many applications, such as in computer games, machinery control (e.g., crane), and thorough mouse replacement. Computer recognition of hand gestures may provide a natural computer interface that allows people to point at or to rotate a computer-aided design model by rotating their hands. Hand gestures can be classified in two categories: static and dynamic. The use of hand gestures as a natural interface serves as a motivating force for research on gesture taxonomy, its representations, and recognition techniques. This paper summarizes the surveys carried out in human-computer interaction (HCI) studies and focuses on different application domains that use hand gestures for efficient interaction. This exploratory survey aims at providing a progress report on static and dynamic hand gesture recognition (i.e., gesture taxonomies, representations, and recognition techniques) in $\mathrm{HCI}$ and to identify future directions on this topic.

As a concluding remark about the new issue of the Journal of Intelligent Industrial systems it can be noted that recent advancements and research findings come to offer reliable solutions to modelling, control and reliability assessment of industrial systems. New control techniques have been developed for industrial systems exhibiting nonlinear dynamics as well as for industrial systems described by PDEs. Besides new approaches have been presented for the assessment of the reliability of industrial systems aiming at enhancing the fault-free functioning of such systems. The presented techniques incorporate intelligence that is capable of treating model uncertainty and imprecise measurements. Thus, the new issue offers a useful insight to the development of intelligent industrial systems.

Dr. G. Rigatos and Dr. P. Siano

Editors-in-Chief of the

Journal of Intelligent Industrial Systems 